\title{
Embedding Integral of a Linear Equation of Degree 3 with One Variable
}

\author{
Rena Eldar Kizi Kerbalayeva ${ }^{1,2}$ \\ ${ }^{1}$ Institute of Mathematics and Mechanics, National Academy of Sciences of Azerbaijan, Baku, Azerbaijan \\ ${ }^{2}$ Middle School №227, Baku, Azerbaijan
}

Email address:

rena-kerbalayeva@mail.ru

\section{To cite this article:}

Rena Eldar Kizi Kerbalayeva. Embedding Integral of a Linear Equation of Degree 3 with One Variable. Mathematics Letters. Vol. 7, No. 3, 2021, pp. 41-44. doi: 10.11648/j.ml.20210703.12

Received: June 14, 2021; Accepted: September 6, 2021; Published: October 30, 2021

\begin{abstract}
What should you able to see this paper? You will see some of calculation estimates and approximations that mathematics can perform as they solve technical problems and communicate their results. The origin of the Linear equation goes back to the early period of development of Mathematics and it is related to the method exhaustion developed by the mathematicians of ancient Babylon and Greece. In this sense, the method of the exhaustion can be regarded as an early method of calculus. The greatest development of method solution of such equation in the early period was obtained in the works Scipione dell Ferro and Niccolo Tartaglia (1539). Systematic approach to the theory of the Linear equation of degree three began in the 16th century. In this paper I studied some properties of the liner equation of degree 3 . The concept of solutions of linear equations is one of most important mathematical concepts. I introduce embedding intervals of the linear equation of degree 3 with one variable. How many solutions are there to the equation? First and foremost, one can discover the definition of a linear equation of degree 3. The Fundamental theorem of Arithmetic says that, the solution of given equation is between divisors of constant term: leading coefficient. But it is very difficult to choose it among them. That is why, we need following condition. Second one can find condition for such equation. The idea that the solution of simultaneous equations $y=x 2, y=(-$ $c x-d):(a x+b)$ is where the graphs intersect can be used too different situations. Your sketching determine the number of real roots of this equation. The solution to this equation are represented by the points where the graphs intersect. Then we can get $\mathrm{y}= \pm(-\mathrm{cx}-\mathrm{d})$ and $\mathrm{y}= \pm(-\mathrm{cx}-\mathrm{d}) 1 / 2 \times(\mathrm{ax}+\mathrm{b}) 1 / 2$. Consider such inequality one can solve by graphical method and by other methods, where $\mathrm{c}, \mathrm{b}, \mathrm{c}, \mathrm{d}$ are real numbers, in addition, $\mathrm{a} \neq-\mathrm{b}: \mathrm{d}$. Here the expression $(-\mathrm{cx}-\mathrm{d}):(\mathrm{ax}+\mathrm{b})$ must be nonnegative. Using it we can find main condition for our given equation. Third I have placed several examples for improved pedagogical format compising the problem's proof, solutions and discussion.
\end{abstract}

Keywords: Linear Equation of Degree 3, Solutions of Equation, Inequality, Graph of Function

\section{Introduction}

In this paper I studied some properties of the liner equation of degree 3. The concept of solutions of linear equations is one of most important mathematical concepts. The original motivation for the linear equation was the problem of the defining solutions of high order equations and calculating the expresiion of such equations. In this paper we will study some basic concepts related to ordinary equation, general solutions of a linear equation of degree three, some method to solve of this equation and some applications of the linear equation of degree three in different types. I introduce embedding intervals of the linear equation of degree 3 with one variable. An equation of degree 3 determines a family of linear equations and one for every value of the constant. Sometimes in attempting to solve a linear equation of degree 3 , we might perform more different steps. This might introduce necessary solutions. If we can get a short list which contains all solutions, then we can test out each one and throw out the invalid ones. The ultimate method is this: does it satisfy given equation? Several methods are used to introduce solutions of this equation. My methods starts with separating given equation into two parts, as undefined concepts and uses them to construct suitable radical inequality, the real numbers, and their solutions. This is an introduction method, which gives us attitude between linear 
equation of degree 3 and rational inequalities. We describe main ideas to solve certain equation due to rational inequality. I introduce new method to find which divisors are solutions of our given equality. In addition, we start our study of linear equations of degree 3 in the same way the pioneers in this field did. Instead of solving these equations people tried to show whether an equation has solutions or not, and what properties such solutions may have. The results of these efforts are shown in the last part of this paper. Often one aim of an exercise is to make students ask questions. They may need help to determine just what the problem is, what constitutes a solution, and where to look for a strategy. Because, it is difficult to choose which of divisors of constant term: leading coefficient are the solutions of this equation. The author generally prefers to see several attempts at most exercises, each one more detailed or progressing father than its predecessors. $[1,3,5]$

\section{Definition and Preliminaries}

In the previous section, we have studied about the linear equations of degree and discussed one method of finding them solutions of such equations. For convenience we give some elementary set definition and terminology. In Algebra we have learnt definition of various linear equations including linear equation of degree 3 . We already familiar with linear equations of degree 3 in one unknown $\mathrm{x}$, which may always be given

$$
a x^{3}+b x^{2}+c x+d=0 .
$$

Where coefficients a, b, c, d for this equation will be considered to be arbitrary real numbers and the coefficient a must be nonzero: $a \neq 0$. It is nice to note that, the solution of this equation is one of the divisor of $\frac{d}{\mathrm{a}}$.

The formulae of elementary Algebra allow us to calculate solutions of many different equations of degree three. However, it is inadequate for calculating the solution of such equations by ordinary Calculus.

Problem in words: it is difficult to choose which of them are the solutions of this equation. That is why I introduce new method for this equation. The solution of this equation can be found as a solution of a linear inequality respectively. In this way such equation can sometime be solved by following method. In this case the problem is reduced to that of solving one inequality of the degree 2 . $[2,4,6]$

Therefore "(1)" becomes in simple form

$$
x^{2}(a x+b)+(c x+d)=0 \text {. }
$$

Then

$$
x^{2}=-\frac{c x+d}{a x+b}
$$

Here the right-hand side of the equation is a linearfractional function and the left member of "(3)" viewed as a quadratic function. Following

$$
x^{2}=y=\frac{-c x-d}{a x+b}=n+\frac{k}{x-m} .
$$

We have studied some linear equations and they solutions by graphical method only, though there many others method also to solve such that problems. The graphical method is of much interest because of their wide applicability in industry, commerce, management science etc. We can sketch graphes of $f \_1=x^{\wedge} 2$ and $f \_2=(-c x-d) /(a x+b)$ both and on the same axes. In this area of study it is convenient and helpful to use geometric terminology. In the two-dimensional Euclidian space, intersections of these functions represent solutions of this equation. If we have one, two, three intersection points then this equation has one, two, three solutions respectively. If it has not any intersection point then it has not any solution. $[7,9,11,16]$

Henceforth we get

$$
x= \pm \sqrt{\frac{-c x-d}{a x+b}}
$$

In addition, one can find the domain of this type of function, just set the term inside the radical sing to $>0$, and solve to find the value that would work for $x$. You cannot take the square root of a negative number, though the radicand to be greater than or equal to 0 . An alternate method to solving the problem is

$$
\frac{-c x-d}{a x+b} \geq 0
$$

The solution set of this inequality is expressed in the form equality "(1)" has solutions within interval satisfying "(6)".

Theorem: Let $a x^{3}+b x^{2}+c x+d=0$ be a given equation. If and if $a d+b c \neq 0$ or $a d-b c \neq 0$ then the equality "(1)" has solutions within interval satisfying “(6)". [8, 10, 12, 14]

\section{Applications}

Below are just some examples of applications of linear equations, that is further applications of this method are given in the exercises.

Examples:

Example 1:

Let $x^{3}-5 x^{2}+16 x-12=0$ be a given equation. It is easy to find solution of this equality: $x=1$. We can verify the condition of our theorem: $1 \times(-12) \neq 5 \times 16$. Then we get

$$
\begin{gathered}
x^{2}(x-5)=-16 x+12 \\
x^{2}=-\frac{16 x-12}{x-5} \\
x= \pm \sqrt{-\frac{16 x-12}{x-5}} .
\end{gathered}
$$

Then we obtain the solution of this inequality: $\left[\frac{3}{4} ; 5\right)$. Indeed $1 \in\left[\frac{3}{4} ; 5\right)$.

Example 2:

Let us to consider the equation $x^{3}-22 x+39=0$. It is easy to verify that $\mathrm{x}=3$ is a solution of our equation.

Formally, one obtains it as follows: 


$$
x= \pm \sqrt{-\frac{39-22 x}{x}}
$$

Here $-\frac{39-22 x}{x} \geq 0$ and we get $(-\infty ; 0) \cup\left[\frac{39}{22} ;+\infty\right)$. Because of $1 \times 39+(-22) \neq 0$. Of course, $3 \in\left[\frac{39}{22} ;+\infty\right)$.

Example 3:

Let us consider $x^{3}+2002 x+2003=0$. We can easily obtain $\mathrm{x}=-1$. One can rewrite given equation as

$$
x= \pm \sqrt{\frac{-2002 x-2003}{x}} .
$$

It has solution $\left[-\frac{2003}{2002} ; 0\right)$. It is clear that $-1 \in\left[-\frac{2003}{2002} ; 0\right)$.

Example 4:

Let $x^{\wedge} 3-5.1 x^{\wedge} 2+4.5 x-0.4=0$ be a given equation. It is easy to find solutions of this equality: $\mathrm{x}=0.1 ; 1 ; 4$. We can verify the condition of our theorem: $1 \times(-0.4)+4.5 \times(-5.1) \neq 0$. Then we get

$$
\begin{gathered}
x^{2}(x-5.1)=-4.5 x+0.4 \\
x^{2}=-\frac{4.5 x-0.4}{x-5.1} \\
x= \pm \sqrt{-\frac{4.5 x-0.4}{x-5.1}}
\end{gathered}
$$

Then we obtain the solution of this inequality: $\left[\frac{4}{45} ; \frac{51}{10}\right)$. Indeed $0.1 ; 1 ; 4 \in\left[\frac{4}{45} ; \frac{51}{10}\right)$.

Example 5:

Let $x^{3}-1.4 x^{2}-1.6 x+0.8=0$ be a given equation. It is easy to find solutions of this equality: $\mathrm{x}=-1 ; 0.4 ; 2$. We can verify the condition of our theorem: $1 \times 0.8+1.4 \times 1.6 \neq 0$. Then we get

$$
\begin{gathered}
x^{2}(x-1.4)=1.6 x-0.8 \\
x^{2}=\frac{1.6 x-0.8}{x-1.4} \\
x= \pm \sqrt{\frac{1.6 x-0.8}{x-1.4}}
\end{gathered}
$$

Then we obtain the solution of this inequality: $(-\infty ; 1.4] \cup[2 ;+\infty)$. Indeed $-1,0.4,2 \in(-\infty ; 1.4] \cup$ $[2 ;+\infty)$.

Example 6:

We knew that, solutions of the equation $x^{3}+3 x^{2}+4 x-$ $12=0$ are numbers $x_{1} \approx 2.1, x_{2} \approx-0.7, x_{3} \approx-0.7$. Consequently,

$$
x= \pm \sqrt{\frac{2}{3-x}}
$$

Following

$$
3-x>0
$$

and $\mathrm{x} \in[-\infty ; 3)$. But one can quickly verify that (according to our condition) $1 \times(-12)+34=0$. Moreover, this equation has no any solutions. $[13,15]$

\section{Conclusions}

The paper provides a transition from elementary calculus to advanced courses in real function theory, linear equation and radical inequality and it introduce the reader to some of the abstract thinking that pervades Linear Algebra. We have studied linear equations of degree 3. Solutions of this equation are closely related to the graph of the given functions. Although these matters are important part of the foundations of mathematics, they have been described in detail here. As a matter of fact, in most phases of Algebra it is only the solution that concern us, rather than the method uses to build to find embedding intervals of solutions of given equation. We also provided some of new exercises. These equations arise in a variety applications, may it be in Physics, Chemistry, Biology, Anthropology, Geology, Economics etc. Hence, an indepth study of such equation has assumed prime importance in all modern scientific investigations.

\section{Acknowledgements}

The author is grateful to the referees for numerous comments that improved the quality of the paper.

\section{References}

[1] Alan S. Tussy., R. David Gustatson., Diane R. Koing.: Basic Mathematics for college students, fourth education, (2011), pp. 594-609, 638-675.

[2] Beezer, R. A. (2015):: A first course in Linear Algebra, University of Puget Sound: Congruent Press, (2015), pp. 1-21.

[3] Connell, E. H. Elements of Abstract and Linear Algebra, Departament of Mathematics University of Miami, (2002), pp $5-13$.

[4] Fabio Cirrito., Nigel Buckle., Iain Dunbar.: Mathematics Higher Level, (2007).

[5] Fine B., Rosenberger G.: The fundamental Theorem of Algebra, Undergraduate texts in Mathematics, Springer-Verlag, New-York, (1997).

[6] Gowers T.: the Princeton Companion to Mathematics (Princeton University Press, 2008).

[7] Jean Linsky., James Nicholson., Brian Western.: Complete Pure Mathematics 213 for Campridge International AS \& Level, (2018).

[8] Jonathan Wicket., Kemper Lewis.: An introduction to Merchanical Engineering, third education, (2013).

[9] Paul Dawkins.: Algebra, (2018).

[10] Sakai T.: Graphs and Trackings (Baifukan, in Japanese, 1963).

[11] Sharipov. R. A.: Course of linear algebra and multidimensional geometry Bashkir State University: Ufa, (1996), pp. 36-38.

[12] Tony Beadsworth.: Complete Additional Mathematics for Campridge IGCSE \& 0level, (2017), pp. 61-62., 92-103. 
[13] Treil, S.: Linear Algebra Done wrong. Department of Mathematics: Brown University, (2014), pp 31-39.

[14] Vaughn Climenhaga.: Lecture notes, Advanced linear Algebra I, (2013).
[15] Takagi T.: Algebra Lecture; Revised New Edition (Kyoritsu Publication, in Japanese, 2007).

[16] Weisstein E. W.: CRC Concise Encyclopedia of Mathematics; English Edution; 2nd Eduation (CRC Press, Kindle version, 1998). 